\title{
DUTY CYCLED MULTI CHANNEL MAC FOR WIRELESS SENSOR NETWORKS
}

\author{
M. Ramakrishnan \\ Department of Electrical and Electronics Engineering, Vel Tech Dr.RR and Dr. \\ SR Technical University, Chennai, India \\ drramakrishnandvel techuniv.edu.in
}

\begin{abstract}
In this work, Duty Cycled Sensor Multi Channel (DC-SMC) Medium Access Control (MAC) has been proposed for wireless sensor networks. The DC-SMC MAC uses a dedicated control channel and multiple data channels. The effective solution for the multi channel hidden terminal problem and missing receiver problem has been proposed in this work. The performance of the $D C$-SMC MAC has been compared with that of the single channel duty cycled CSMA/CA MAC by taking the throughput and latency as performance metrics. It has been shown that the duty cycled multi channel MAC gives high throughput and less latency even with lower duty cycles.
\end{abstract}

\section{KEYWORDS}

Multi Channel Medium Access Control, Duty Cycling, Wireless Sensor Networks, Multi channel hidden terminal problem

\section{INTRODUCTION}

A Wireless Sensor Network is the network of tiny devices, which has both sensing and communication capabilities. Nowadays, many sensor network hardware platforms like MICAZ, Telos, etc, have an RF transceiver which is capable of communicating in different channels which can be dynamically selected from the firmware. The multi channel capability gives another degree of freedom for medium access in wireless sensor networks. Still multi channel medium access control inherently has some issues which have to be dealt with carefully, while doing the MAC design to improve the network performance. We classify the multi channel MAC protocols according to the channel assignment methods: fixed assignment, semi-dynamic assignment and dynamic assignment. In fixed assignment approaches, the radios are assigned channels for permanent use. Although the assignment of the channels can be renewed, for instance due to changing interference conditions, radios do not change the operating frequency during communication. In semi-dynamic approaches, the radios are assigned constant channels, either for receiving or transmitting, but it is possible to change the channel for communicating with the radios that are assigned different channels. In dynamic approaches, nodes are not assigned static channels and can dynamically switch their interfaces from one channel to another between successive data transmissions.

Natarajan Meghanathan et al. (Eds) : ICCSEA, SPPR, VLSI, WiMoA, SCAI, CNSA, WeST - 2014

pp. 157-174, 2014. (O) CS \& IT-CSCP 2014

DOI : $10.5121 /$ csit.2014.4723 
In dynamic channel assignment approaches, every data transmission takes place after a channel selection. The channel selection can be, measurement based or status based. In measurement based approaches, the communicating parties measure the SINR values on a channel before transmitting. In status-based approaches, the nodes keep track of the status of the channels, such as busy or idle, according to the received control packets. In the dynamic multi channel MAC design, there are three types of implementations. They are, the split phase, the dedicated control channel and the channel hopping. In the Dedicated Control Channel based multi channel MAC, the nodes synchronize by exchanging control packets on the dedicated control channel and negotiate for the channel to be used for data exchanges. Examples of the dedicated control channel approaches are presented [1][2]. In the split phase approach, such as the MMAC [3] and MAP [4] time is divided into two phases - the appointed phase and the data transmission phase. In the appointed phase the nodes negotiate and select their channel for communication through the exchange of control packets in a common channel. In the data transmission phase, the data packets are transmitted on selected channels. This split phase multi channel MAC requires time synchronization among nodes. In the frequency-hopping approaches, nodes switch, or in other words hop, between different channels.

In the literature, the multi channel MAC has been proposed for systems with multiple transceivers and a single transceiver. To keep the cost and power consumption low, sensor nodes are equipped with a single transceiver. In this work a multi channel MAC has been proposed for wireless sensor nodes with a single half duplex transceiver, which uses a dedicated control channel for channel negotiation. A multi channel protocol performs better in the one-to-one topology, rather than in a star topology or topologies in which multiple source nodes communicate with a single sink node. But in sensor networks, convergecast communication is often used where multiple sensor nodes report their sensor data to the base station either in a single hop or multi hop fashion. So, the advantages of using multiple parallel links for communication shrinks, when the packet converges towards the base station in multi hop networks. To avoid this, multiple radios can be used at the Base Station to exploit the power of multi channel communication in Convergecast communication also. In general, in an ad hoc wireless sensor network, the multi channel MAC protocol improves the throughput and latency performance, as it allows concurrent transmissions in different orthogonal channels. This kind of multi channel MAC is inherently suitable for the Wireless Network Control System (WNCS), where the Multi channel MAC makes many wireless control loops co exist with each other. This significantly improves the network delay, which is the major influencing factor in the system performance in WNCS.

The paper is organized as follows. Section 2 describes the multi channel MAC proposed in the literature and section 3 discusses the proposed sensor multi channel MAC and its simulation results. Section 4 discusses the MATLAB based discrete event simulation of the proposed Sensor Multi Channel (SMC) MAC in a multi hop scenario, and section 5 reports the proposal of the duty cycled Sensor multi channel MAC (DC-SMC).

\section{RELATED WORK}

In this work, the usage of the multi channel MAC is to eliminate the interference to give better performance in terms of throughput and latency. Also the multi channel capability is exploited to give better energy efficiency in wireless sensor networks. This is achieved by introducing the multi channel feature in duty cycled MAC protocols to keep the throughput and latency constant even in low duty cycle conditions. In some works, the multi channel capability is used to avoid 
jamming attacks [5][6][7]. Reference [8] has proposed a TDMA based multi channel MAC, YMAC for wireless sensor networks. It requires time synchronization among nodes. Reference [9] has proposed HyMAC, a hybrid TDMA/FDMA Medium Access Control for wireless sensor networks. It schedules the medium access for the nodes while using the multiple frequencies available in the commercial sensor node hardware platforms. In a reference [10], a TDMA based multi channel MAC for wireless sensor networks, TFMAC, has been proposed. The TFMAC requires time synchronization, and it uses single half duplex transceiver. This protocol divides each channel into time slots and the slot scheduling has been done in the medium access. The frame has been divided into a contention access period where the slot scheduling and channel allocation has been done and a contention-free period where the data transfer has been done. In the literature, a Multiple frequency Medium access control for wireless Sensor Networks (MMSN) [11] has been proposed, which divides the protocol into two functionalities. They are frequency assignment and medium access. In the frequency assignment, four different techniques are proposed. They are, 1. Exclusive frequency assignment 2. Even selection 3. Eavesdropping 4. Implicit consensus and the medium access is done by dividing the frame into broadcast contention period $\left(\mathrm{T}_{\mathrm{bc}}\right)$ and transmission period $\left(\mathrm{T}_{\mathrm{Tran}}\right)$. The node contents for the channel, for both broadcast and unicast with a non uniform back off. The paper assumes that the nodes are stationary, time synchronized and the frequency assignment has been done once. The Time synchronization overhead becomes higher than the RTS/CTS control packet overhead during low traffic conditions. Moreover, maintaining a tight time synchronization in the ad hoc multi hop wireless sensor is difficult. Hence, in this work, a dedicated control channel based multi channel MAC has been proposed for the wireless sensor network. In reference [12] asynchronous multi channel protocol (AMCP) has been proposed. The two issues, information asymmetry and flow in the middle which happens while using CSMA/CA in multihop environment has been stated. The multi channel MAC issues such as multi channel hidden terminal problem and missing receiver problem also has been stated. And the bottleneck analysis of dedicated control channel also reported and the theoretical upper bound for the number of data channels for a given channel capacity has been given. Though SMC MAC design has been inspired from the AMCP [12], the following differences exist.

1. In the channel negotiation of the AMCP, the transmitter selects a free channel and sends RTS with the selected channel. If the channel is not available in the receiver, it sends the negative CTS (nCTS) along with its channel status. Then the transmitter selects a common free channel as its preferred channel. This channel selection procedure decreases the throughput and latency performance, if the occurrences of transmission of nCTS are many. To avoid this, in the proposed SMC MAC, the transmitter sends its entire channel status to the receiver and the receiver selects a free common channel.

2. The AMCP does not explicitly specify the overhead associated with channel negotiation, whereas in the SMC MAC only an 8 bit field (Channel Status) has been included with the RTS and CTS control packet. This reduces the control packet overhead in wireless sensor networks where the data packet size is small.

3. The AMCP handles the Multi Channel Hidden Terminal (MCHT) problem by making the transmitting node wait after transmission for a specific time to avoid collision due to loss of channel information. This increases the latency. In the SMC MAC, the transmitting node senses the other channels to regain the information about other data channels. Hence the latency performance can be retained. 
4. In the SMC MAC, the control channel is used as a broadcast channel which is required for many routing protocols in the network layer.

In a reference [13] the performance evaluation of the multi channel extension of 802.11 MAC has been done. It is stated that the channel assignment can be done by the measurement based method and the status based method. In our proposed multi channel MAC protocol, we use the status based method for channel assignment, and the measurement based method for avoiding loss of channel information problem, which is prevalent in the dedicated control channel multi channel medium access control protocols. In a reference [14], a cooperative multi channel MAC (CAM MAC) has been proposed, in which the loss of channel information problem can be solved by getting the channel information from the cooperating neighbouring node to select a collision free channel for the communicating nodes. In another reference[15], the On Demand Channel Switching (ODC) has been proposed for multi channel medium access control. In this protocol, each node will stay in a channel as long as its traffic share in that channel does not go below a threshold value. If the traffic share of the node in a channel goes below the threshold, then the node will switch to a different channel after broadcasting the switching event. In a reference [16], the signal strength measurement based channel selection has been done in the proposed multi channel CSMA MAC. In reference[3], So et al have proposed the MMAC, which is a split phase multi channel medium access control protocol. At the starting of the beacon interval in the ATIM window, the node which has packets to transmit will negotiate for the channel, and if the channel is acquired, then the communicating nodes switch to that data channel and do the data transfer.

\section{Sensor Multi ChANNEL MEDiUM ACCESS CONTROL FOR WIRELESS SENSOR NETWORKS}

\subsection{SMC MAC Algorithm}

The proposed Multi channel MAC, Sensor Multi Channel MAC, has been described in the following section. The SMC MAC uses a single dedicated control channel and eight data channels. The Multi Channel MAC has been designed by taking the following points into consideration.

All the nodes are equipped with a single half duplex transceiver, which has the capability to switch from one channel to another channel dynamically. The switching can be done via software control. There are eight data channels and one control channel, and all the channels have equal capacity. The channel switching time is assumed to be negligible and all the channels are orthogonal and non-overlapping.

As a single channel is dedicated to the control packet flow, it creates a bottleneck. It poses a constraint on the number of data channels that can be used in the multi channel MAC. The number of data channels that can be used in a dedicated control channel MAC, is given by the following expression by neglecting the back off time.

$$
M=\frac{\left(T_{D}+T_{R}+T_{C}\right)}{T_{R}+T_{C}}
$$




\begin{tabular}{|c|c|c|c|}
\hline where & $\mathrm{T}_{\mathrm{D}}$ & - & DATA and ACK transfer period \\
\hline & $\mathrm{T}_{\mathrm{R}}$ & - & RTS packet transfer period \\
\hline & $\mathrm{T}_{\mathrm{C}}$ & - & CTS packet transfer period \\
\hline & $\mathrm{M}$ & - & Maximum Number of data channels \\
\hline
\end{tabular}

If the packet transfer period is quantified in bytes for convenience, then the $T_{D}, T_{R}$ and $T_{C}$ in the proposed SMC MAC protocol are 107(100+7), 7 and 7 bytes respectively. By substituting this we get $\mathrm{M}=8$.

The SMC MAC is described as follows:

1. Initially all the nodes stay in the control channel. The channel negotiation is done via the RTS/CTS control packets

2. When a packet arrives in a node, it sends the RTS with its channel status. The channel status is an eight bit field in which 0 indicates a free channel and 1 indicates a busy channel.

3. When the node to which the RTS has been transmitted, receives this packet, it selects the first common free channel for both the transmitter and the receiver and intimates the selection by setting the corresponding bit in the channel status field. Then the CTS is transmitted through this channel status field.

4. After the transmission of the CTS packet, the transmitting node switches to the selected channel

5. When the CTS packet is received by the intended node, it switches to the selected data channel. When the CTS is received by unintended nodes, the selected channel is marked as busy in the channel status table. The channel is marked as an idle channel, after the DATA+ACK transfer period.

6. The DATA and ACK are transmitted in the data channel. After the transmission and reception of the ACK packet, the node measures the RSSI for each channel and updates the channel status table. Thus, the loss of channel information problem is countered. Then, the node switches to the control channel. 


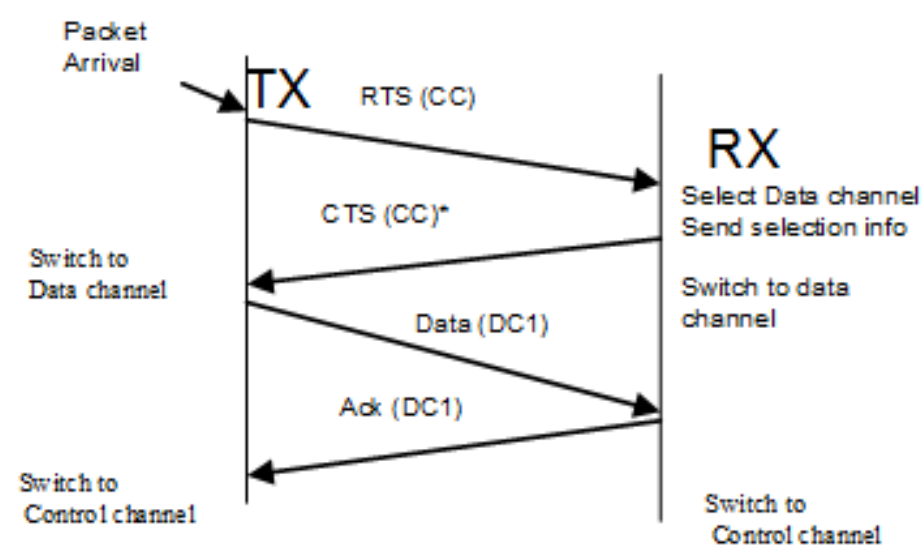

(a)

CHANNEL NEGOTIATION - Algorithm

1: $\quad$ Node.Ch_Status $=[0,0,0,0,0,0,0,0,0]$

2: $\quad$ IF Unicast Packet Arrival THEN

3: $\quad$ RTS.Ch_Status $=$ Node.Ch_Status

4: $\quad$ Send Request To Send (RTS) Packet

5: $\quad$ END IF

6: IF RTS Received THEN

7: $\quad$ flag $=0$;

8: $\quad$ FOR $k=1: 8$

9:

IF RTS.Ch_Status $[k]==0$ AND

Node.ch_status $[k]==0$ THEN

10 :

Node.Ch_status $[k]=1$;

11:

CTS.Ch_status $[k]=1$;

12

CTS.Active_Channel $=k$;

13:

Node.Active_Channel $=k$;

14:

flag=1;

15:

break;

16:

END IF

17:

END FOR

18 :

IF flag $=0$ THEN

19:

--No Common free channel is available

20:

Do Nothing; 


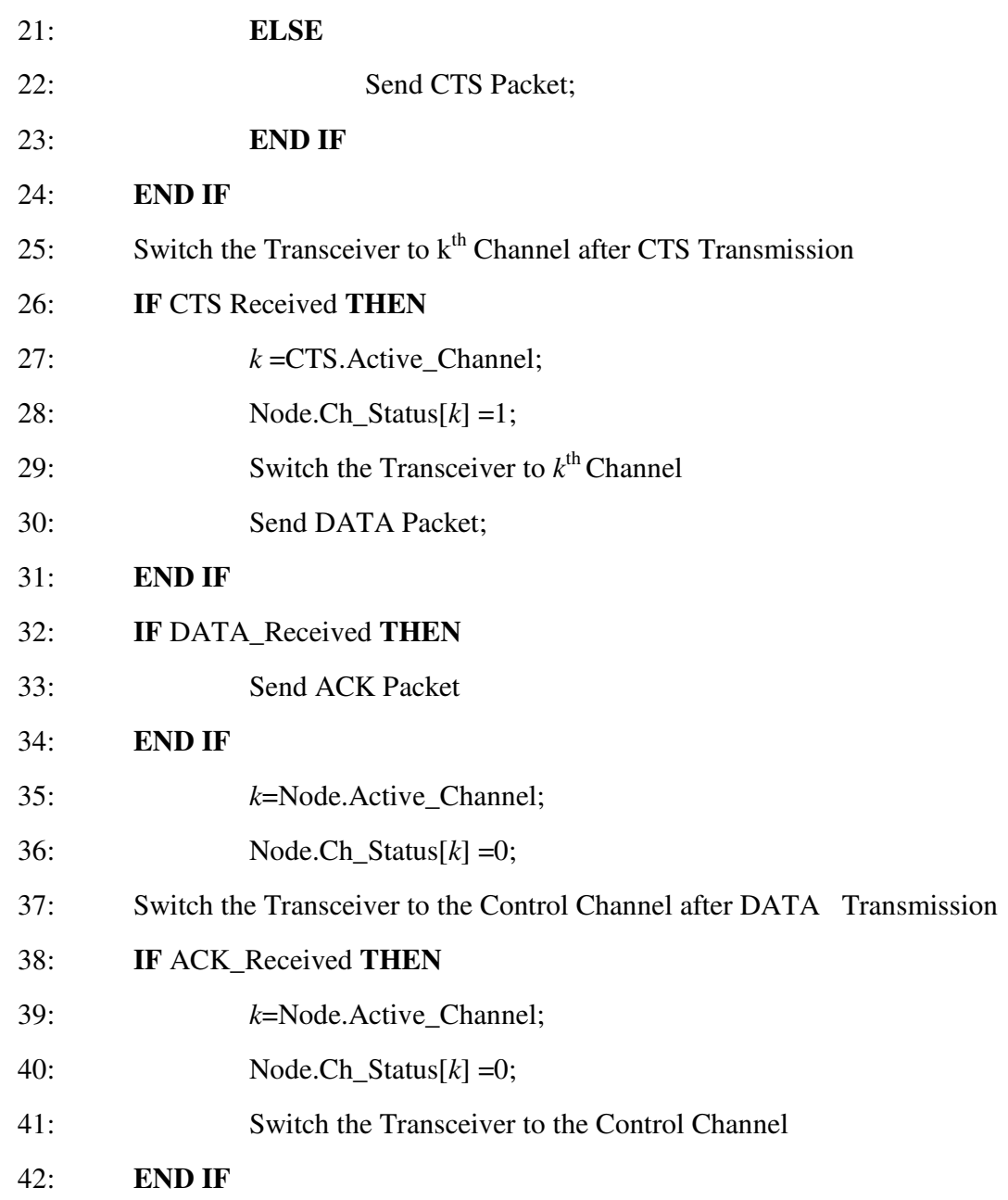

(b)

Figure 1.(a) Unicast packet flow in SMC MAC (b) Channel Negotiation Algorithm

The Control channel is used as the broadcast channel to support the broadcast which is required for the route discovery process of some routing protocols. Figure 1 illustrates the channel assignment and data transfer in SMC MAC.

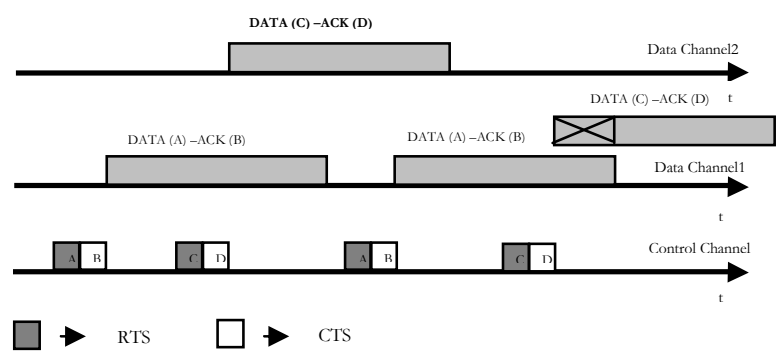

Figure 2. Multi Channel Hidden terminal problem 
In the scenario shown in Figure 2, during the $\mathrm{CD}$ pair's data communication at channel 2, the $\mathrm{AB}$ pair has selected channel 1 for its data communication through the RTS/CTS packet $\left(3^{\text {rd }}\right.$ control packet in Figure 2). Now as the CD pair could not overhear the channel negotiation of the $A B$ pair, it has lost the channel 1 information. When the next unicast packet arrives at node $\mathrm{C}$, the node selects data channel 1 which is already in use by the AB pair. This causes a collision. This is called the Multi Channel Hidden Terminal Problem. This happens due to the loss of channel information for the nodes $\mathrm{C}$ and $\mathrm{D}$.

The SMC MAC solves this problem by sensing all the channels after the data transfer as shown in Figure 3. In the missing terminal problem shown in Figure 4, the node A tries to communicate with node $\mathrm{C}$ by sending the RTS packet, while $\mathrm{C}$ is busy in data transfer at channel 2 . This problem can be alleviated in the SMC MAC by increasing the RTS timeout value, when the transmitter has detected that some data channel has gone into the busy state, while it was doing its previous data transfer. The RTS timeout happens in the wireless environment, due to a low SNR for the RTS in the receiver. In the multi channel environment, as the data transfer is offloaded from the control channel, the probability of getting high noise (low SNR) in the control channel is low.

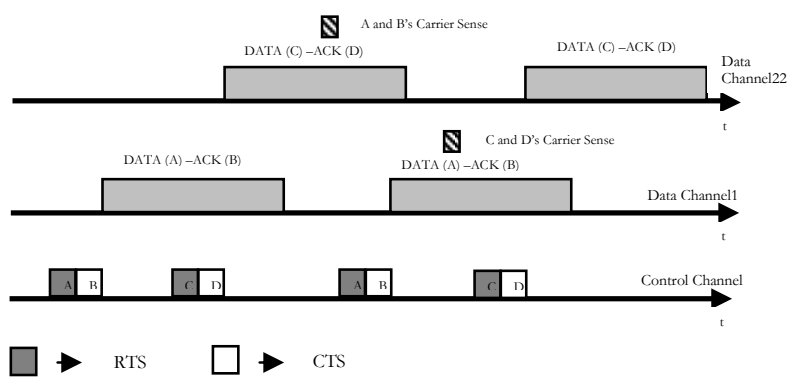

Figure 3. SMC MAC solution for multi channel hidden terminal problem

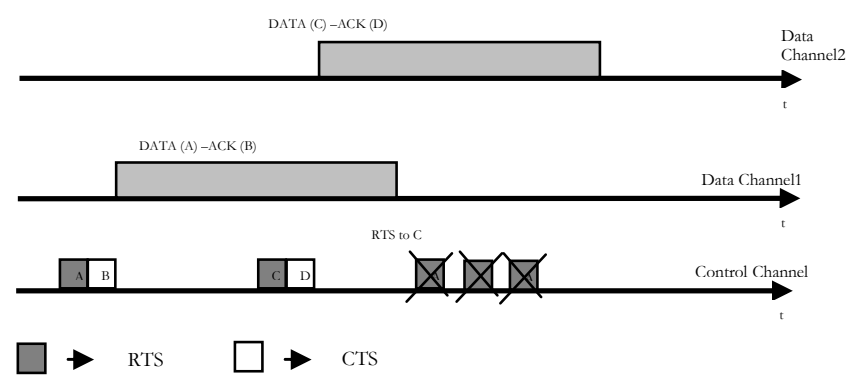

Figure 4. Missing Terminal Problem

\subsection{Simulation of the SMC MAC:}

A discrete event simulation has been done in MATLAB to analyze the performance of the proposed multi channel MAC. The simulation has been done for the single hop topology. In a single hop environment nodes are placed at random, and the one-to-one traffic is given. The simulation is repeated for different packet inter arrival times. Through simulation, the 
performance metrics, the throughput and latency of the single channel MAC have been compared with those of the SMC MAC.

Table 1. Channel Status Table

\begin{tabular}{|l|l|l|l|l|l|l|l|l|}
\hline & Ch1 & Ch2 & Ch3 & Ch4 & Ch5 & Ch6 & Ch7 & Ch8 \\
\hline Status & 0 & 1 & 0 & 0 & 0 & 0 & 1 & 1 \\
\hline Tx & - & 2 & - & - & - & - & 4 & 1 \\
\hline Rx & - & 3 & - & - & - & - & 5 & 10 \\
\hline
\end{tabular}

0 - IDLE channel; 1 -BUSY channel

In the physical layer, a log-shadowing radio model is used. CSMA/CA with the RTS/CTS and random back off mechanism has been used in the MAC layer. Each node has a channel status table, which has a structure, shown in Table 1. The simulation parameters are summarized in Table 2.

Table 2. Simulation Parameters

\begin{tabular}{|c|c|c|}
\hline S.No & Simulation Parameters & Value \\
\hline 1. & Number of Data Channels & 8 \\
\hline 2. & Radio Model & Log-Shadowing Model \\
\hline 3. & MAC Layer & $\begin{array}{l}\text { CSMA/CA with RTS/CTS and } \\
\text { Multi Channel extension }\end{array}$ \\
\hline 4. & Data rate & $115 \mathrm{kbps}$ \\
\hline 5. & Max. Power & $+13 \mathrm{dBm}$ \\
\hline 6. & Area & $30 \times 30 \mathrm{~m}$ \\
\hline 7. & Topology & Single hop Random Topology \\
\hline 8. & $\mathrm{SNR}_{\text {threshold }}$ & $+30 \mathrm{dBm}$ \\
\hline 9. & $\begin{array}{l}\text { Size of packets } \\
\text { RTS/CTS/DATA/ACK }\end{array}$ & 7/7/100/7 Bytes \\
\hline
\end{tabular}




\subsection{Performance of the Proposed Multi Channel MAC:}

Simulation has been repeated for different values of the packet inter arrival time and the network throughput and latency have been observed for various traffic loads. From Figure 5 it is observed, that the throughput of the proposed multi channel MAC is higher than that of the single channel MAC during high traffic conditions. When there is a light traffic load, the performance of the multi channel and single channel MAC are similar. It is observed from Figure 6 that the latency in high traffic conditions for the SMC MAC is lower than that of single channel MAC. In this protocol, a random back off scheme is implemented for the access of the control channel. The contention in the control channel limits the latency performance of the SMC protocol in high traffic.

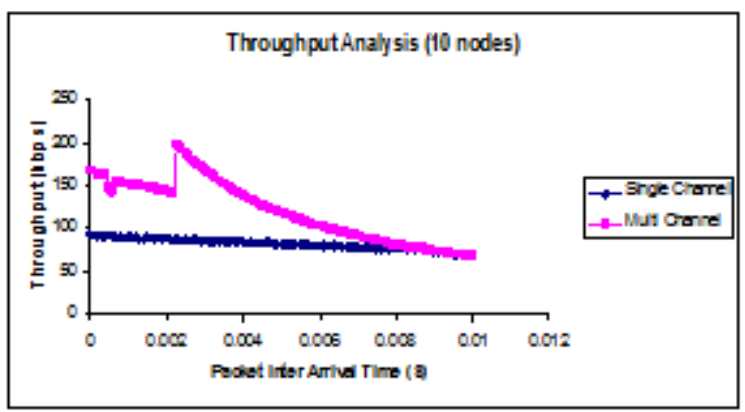

(a)

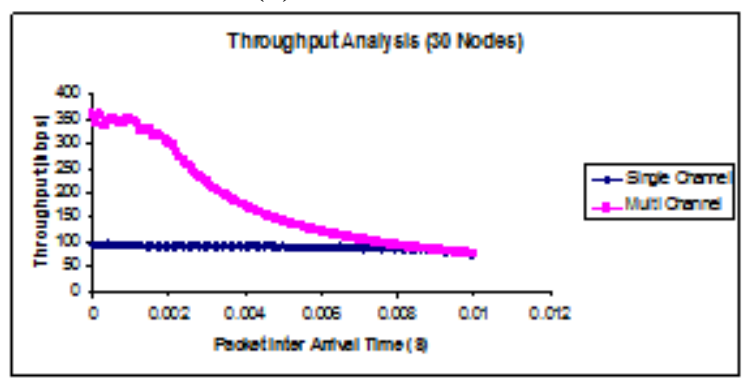

(c)

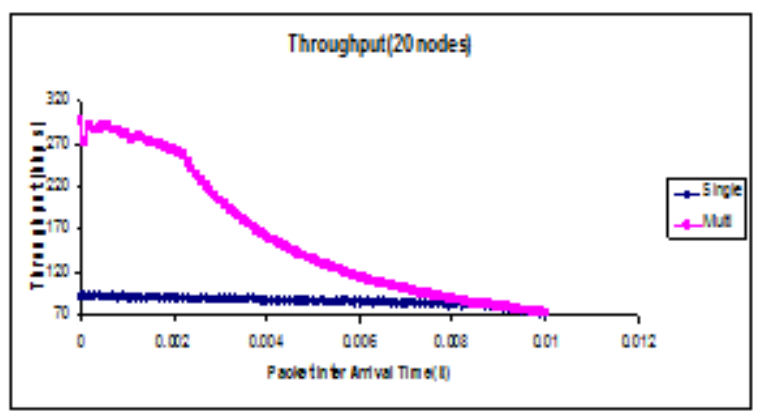

(b)

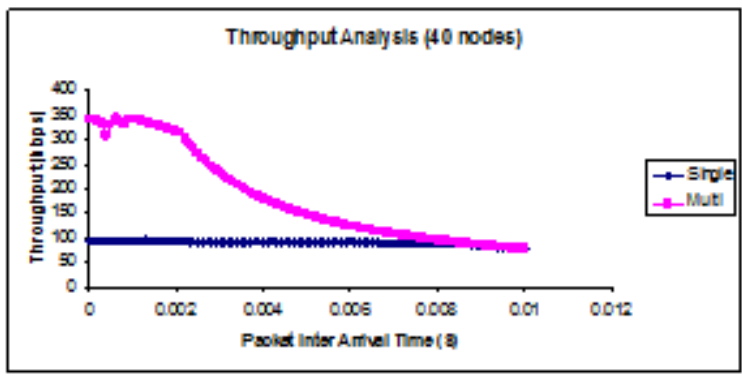

(d)

Figure 5 Throughput Analysis for Various Node densities (Random Topology) (a) 10 nodes (b) 20 nodes (c) 30 nodes (d) 40 nodes

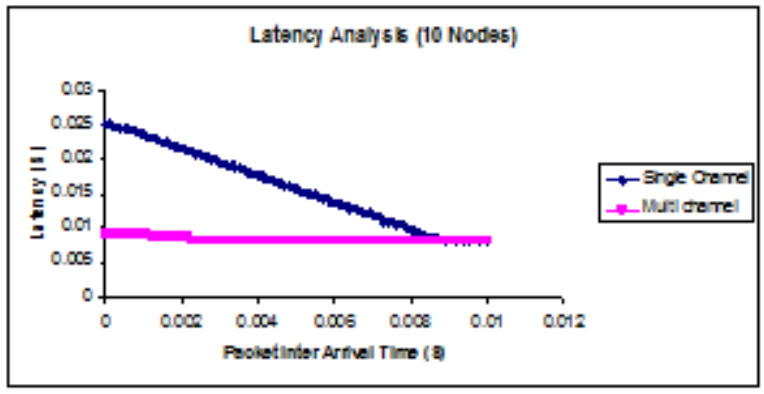

(a)

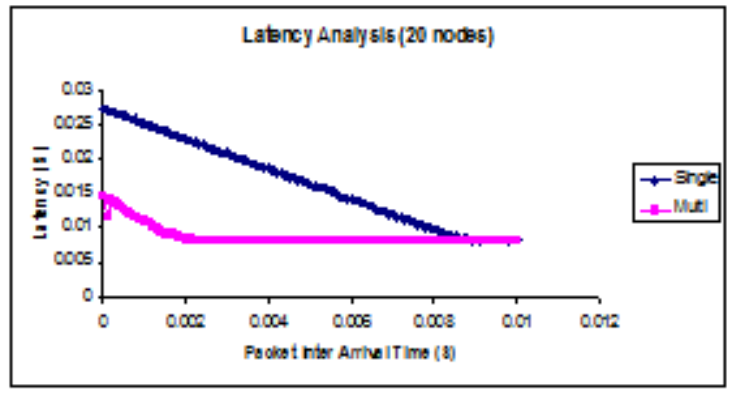

(b) 


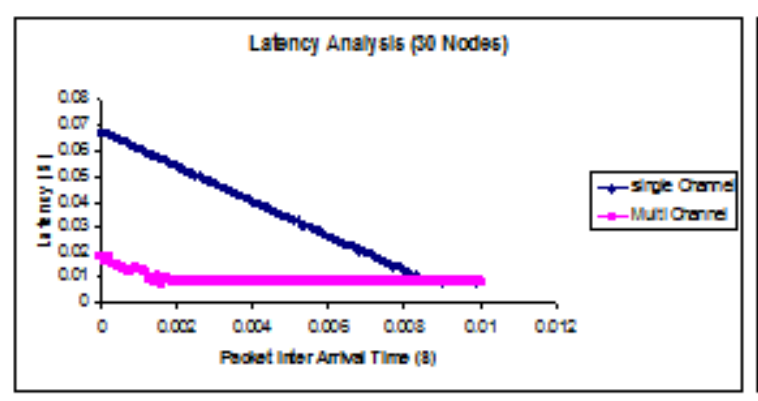

(c)

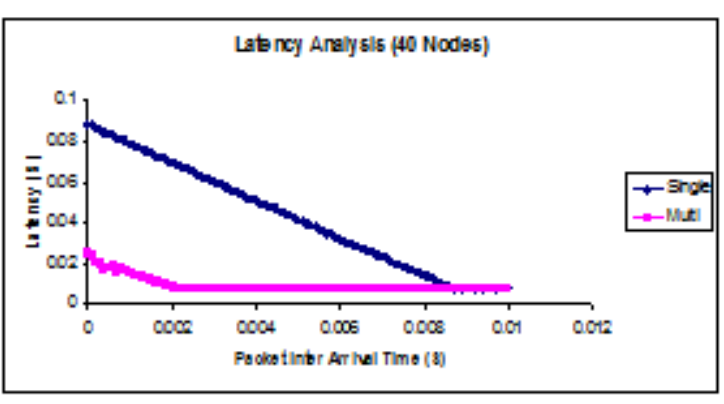

(d)

Figure 6. Latency Analysis for various Node Densities (Random Topologies) (a) 10 nodes (b) 20 nodes (c) 30 nodes (d) 40 nodes

Figure 7 shows the effect of the Multi Channel Hidden Terminal (MCHT) Problem on the throughput. Due to the loss of channel information, nodes select the data channel which is busy. This causes the collision of data packets, which decreases the throughput significantly. From Figure 7, it is observed that carrier sensing to retrieve channel information helps avoiding the Multi channel Hidden Terminal Problem in the proposed Multi Channel MAC.

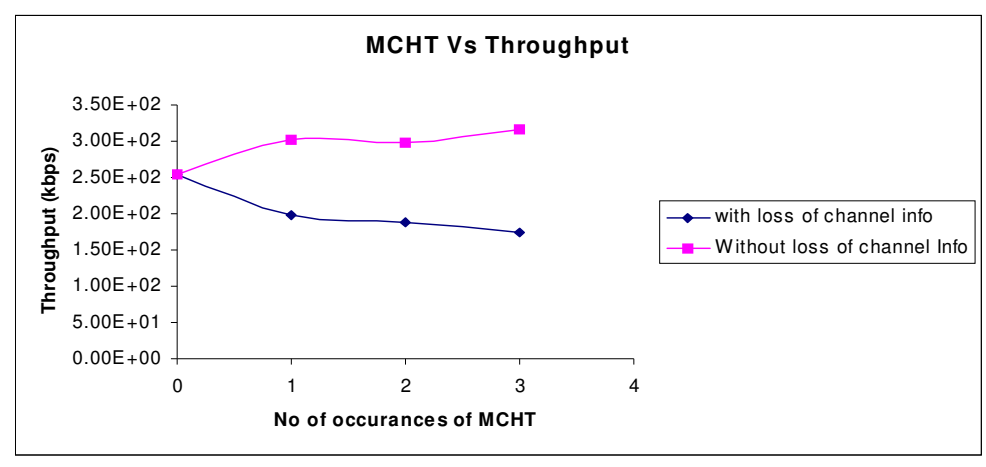

Figure 7. Effect of the Multi Channel Hidden terminal Problem on Throughput

\subsection{Comparison of the SMC MAC with the AMCP}

In the AMCP protocol channel negotiation is done as mentioned below.

The transmitter node, for the unicast packet should select a particular free channel as the preferred channel. And the RTS is transmitted with the preferred channel field. Now the receiver checks whether the preferred data channel is free. If it is free, then the CTS is sent, and it changes to the preferred data channel. After getting the CTS, the transmitter changes the frequency channel to the preferred channel, and transmits the DATA packet. If the preferred channel is busy in the receiver, then the receiver transmits a negative CTS (nCTS) packet along with its channel status. After receiving the nCTS the transmitter once again selects the common free channel, as the preferred channel and the RTS is transmitted again. When the preferred channel is not available in receiver, two extra control packets (nCTS and RTS) have to be transmitted. This affects the throughput and latency performance of the AMCP. To avoid the multi channel hidden terminal problem, after the data transmission, the AMCP marks the status of all the channels except the 
current data channel as busy for the transmitter and receiver. The simulation has been done for the sensor network with 40 nodes, which are arranged in a single hop random topology to compare the performance of the SMC and the AMCP MAC in terms of throughput and latency. Figures 8 shows the throughput and latency comparison of the SMC and the AMCP MAC.

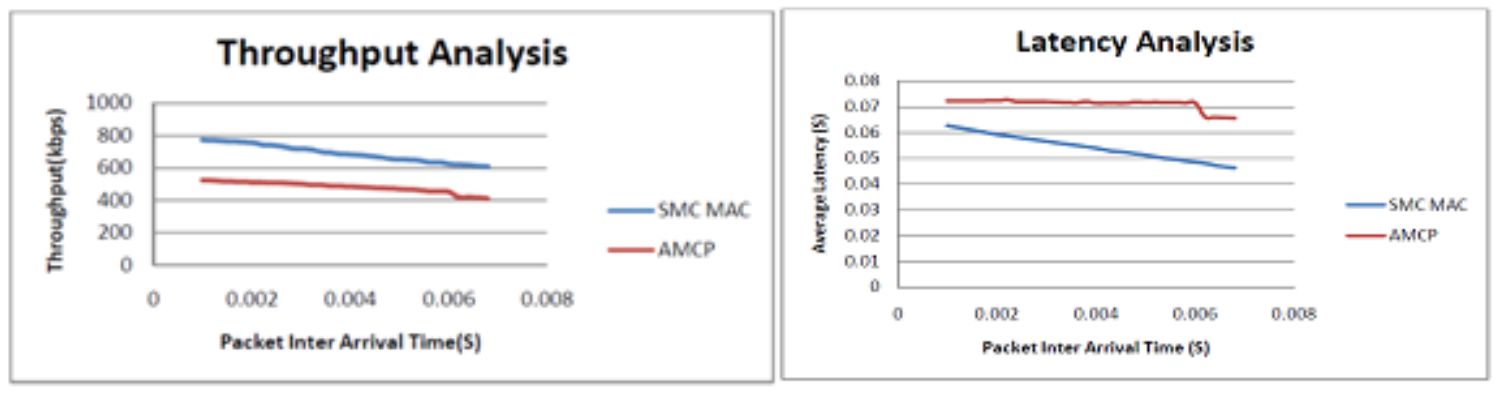

Figure 8. Throughput and Latency of SMC and AMCP MAC

From Figure 8, it is shown that the throughput of the SMC MAC is $27 \%$ to $32 \%$ higher than that of the AMCP. From the results it is shown that the throughput of the SMC MAC is $13 \%$ to $32 \%$ lower than the latency of the AMCP. The reason for this performance difference is that the AMCP inhibits the use of the data channels after the data transmission and reception. This leads to a negative CTS flow, and a consecutive RTS flow. This causes the drop in latency and throughput performance, whereas in SMC MAC, the RTS is transmitted with the channel status, so the receiver finds the common free channel and selects that for the data communication. To solve the Multi channel hidden terminal problem the AMCP proposes a method of inhibiting the nodes from using the channels other than the current data channel used Figure 10 shows the effect of Multi Channel Hidden Terminal Problem(MCHT) occurrences in throughput performance of SMC and AMCP MAC. The throughput of the multi channel MAC without MCHT avoidance is also shown.

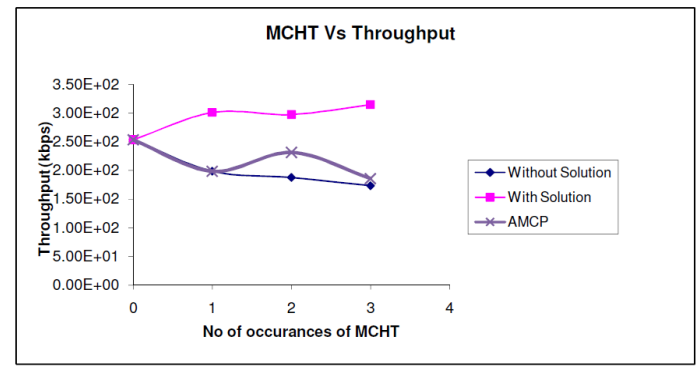

Figure 10. Impact of MCHT on Throughput of SMC and AMCP MAC

\section{SMC-MAC SIMULATION IN MULTIHOP SCENARIO:}

The low power radio in a wireless sensor node limits the communication range in single hop topologies. So it is preferable to have multi hop communication in wireless sensor networks. 
Table 3. Simulation Parameters (Multi hop Scenario)

\begin{tabular}{|l|l|l|}
\hline $\begin{array}{l}\text { S.N } \\
\text {. }\end{array}$ & Simulation Parameters & Value \\
\hline 1. & Number of Data Channels & 8 \\
\hline 2. & Radio Model & Log-Shadowing Model \\
\hline 3. & MAC Layer & $\begin{array}{l}\text { CSMA/CA with RTS/CTS and } \\
\text { Multi Channel extension }\end{array}$ \\
\hline 4. & Data rate & $115 \mathrm{kbps}$ \\
\hline 5. & Max. Power & $+13 \mathrm{dBm}$ \\
\hline 6. & Area & $50 x 50 \mathrm{~m}$ \\
\hline 7. & Topology & $+30 \mathrm{dBm}$ \\
\hline 8. & SNR threshold & $7 / 7 / 100 / 7$ Bytes \\
\hline 9. & $\begin{array}{l}\text { Size of packets } \\
\text { RTS/CTS/DATA/ACK }\end{array}$ & DSR \\
\hline 10 & Routing Protocol & \multicolumn{2}{|c|}{} \\
\hline
\end{tabular}

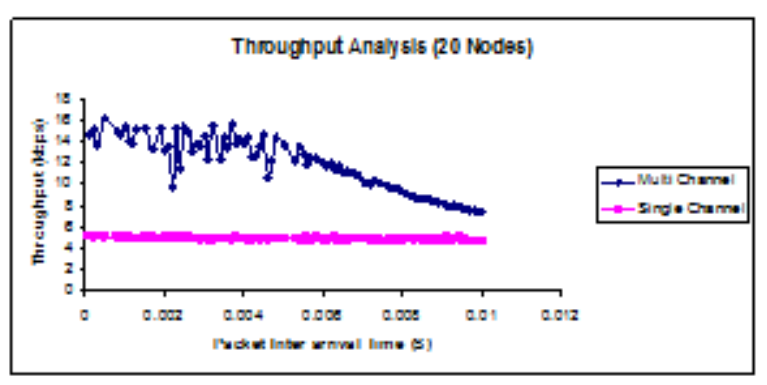

(a)

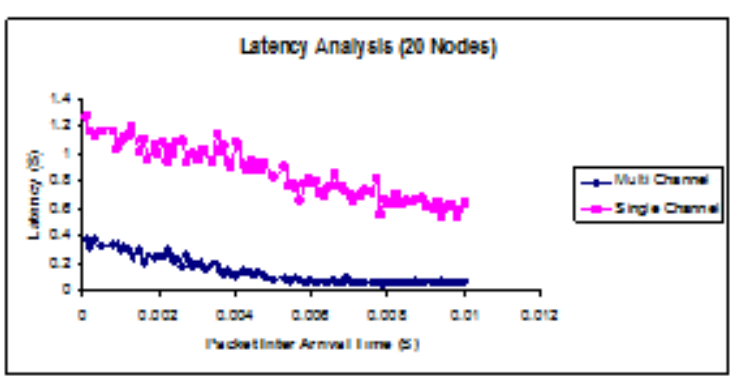

(b)

Figure 11.Performance Comparison of SMC MAC with Single Channel MAC in Multi hop environment (a) Throughput Analysis (b) Latency Analysis

The proposed SMC MAC has been simulated in MATLAB with multi hop random topologies. Dynamic Source Routing (DSR) is used as the routing protocol. The throughput and latency have been taken as performance metrics and simulation has been done for multiple node densities. The simulation parameters are summarized in Table 3. DSR is a reactive routing protocol. It will search for the route by broadcasting the Route Request (RREQ) packet. The propagation of the RREQ packets diminish the throughput and latency performance of the multi hop network more than that of single hop network topologies. From Figure 11(a) it is observed that the throughput performance of the SMC MAC is up to $70 \%$ higher than that of Single Channel CSMA/CA MAC. From Figure 11(b) it is observed that the latency performance of SMC MAC is up to 91\% lower than its single channel counterpart. In the multi hop environment, the RREQ packet 
propagation as broadcast in the network inhibits the greater throughput performance of a multi channel MAC. The higher throughput performance of the multi channel MAC is attributed to the parallel unicast packet flow in high traffic conditions.

\section{DUTY CyCLED MULTi ChANNEL MAC}

\subsection{DC-SMC MAC}

In a typical Medium Access Control for Wireless Sensor Network, duty cycling has been adapted for the energy conservation in a battery powered sensor node. The node periodically goes to sleep and to the listen state. The energy consumption of the 802.11 MAC is 2-6 times that of the SMAC. Still the energy conservation due to the periodic sleep/wakeup schedule comes at the cost of increased latency and decreased throughput. In an Event driven sensor network, though the network is idle most of the time, when the event occurs the traffic will be significantly more. In this case, the duty cycled MAC exhibits increased latency and decreased throughput. In this work to reduce the latency, with the fixed duty cycle MAC, multi channel capability is combined, which significantly improves the latency performance even if the sleep time is more.

The periodic sleep/wakeup schedule has been included with the sensor Multi Channel MAC. The duration of the period $\left(\mathrm{T}_{\mathrm{ON}}+\mathrm{T}_{\mathrm{OFF}}\right)$ is set as $6 \mathrm{~s}$ and for various duty cycles $4 \%, 6 \%, 8 \%, 10 \%$ and $12 \%$, the network throughput and average packet latency have been derived from the MATLAB based discrete event simulation. In simulation the one-to-one traffic is taken to show the performance of the Duty Cycled Multi Channel MAC. The data rate has been set as 9.6kbps and the size of the data packet is 100 bytes.

The simulation has been repeated by varying the packet inter arrival time, which is varied till half the period duration (3s). When the packet arrives at the MAC layer, two conditions are checked to process it. The conditions are

1. Whether the node is in the wakeup state? If the node is in the sleep state, then the packet has to be buffered for the transmission at the next wakeup schedule. If the node is in the wakeup state then the next condition is checked.

2. Whether the duration of the transmission of the packet is within the wakeup period.

If both the conditions are satisfied then the packet will be transmitted otherwise the packet is buffered for transmission at the next wakeup schedule.

\subsection{RESULTS AND DISCUSSION}

An analysis of the network throughput and average packet latency has been done for various duty cycle values $(4 \%, 6 \%, 8 \%, 10 \%$ and $12 \%)$. As the number of packets transmitted is constant, the performance of the DC-SMC MAC becomes similar to that of the single channel duty cycled MAC, when the duty cycle increases. Figures 12 and 13 show the throughput and latency performance of the DC-SMC MAC and the single channel duty cycled MAC. From Figure 14, it is observed that the throughput of the DC-SMC MAC outperforms that of the single channel duty cycled MAC, during the lower duty cycle conditions which is necessary for the energy conservation. When the duty cycle is increased to $12 \%$ (Figure 12d.) the throughput performance 
of DC-SMC MAC is similar to that of single channel MAC as there is no accumulation of packets to send as a burst at the starting of the wakeup period. Similarly, Figure 13 shows the variation of the latency for the different packets inter arrival time. It is also observed that the latency performance of the DC-SMC MAC is almost unaltered by the duty cycle variation for a particular traffic. Figure 14 shows the variation of the throughput and average packet latency for different duty cycle values. From Figure 14 it is observed that the throughput and latency of the DC-SMC MAC shows almost no variation for different duty cycle values, whereas the variation of the throughput and latency of the duty cycled single channel MAC is significant for different duty cycle values. As the duty cycle is directly proportional to the energy consumption, with the DCSMC MAC, a higher throughput and lower latency can be achieved with minimal energy consumption.

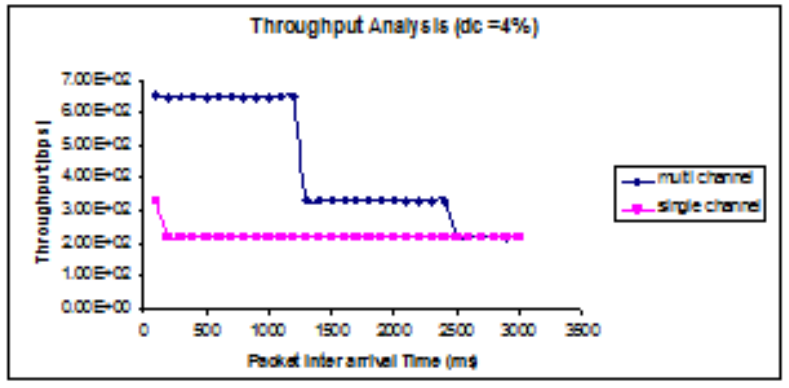

(a)

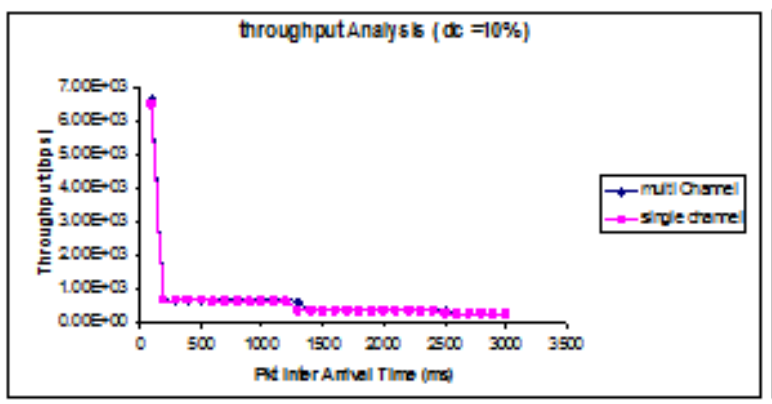

(c)

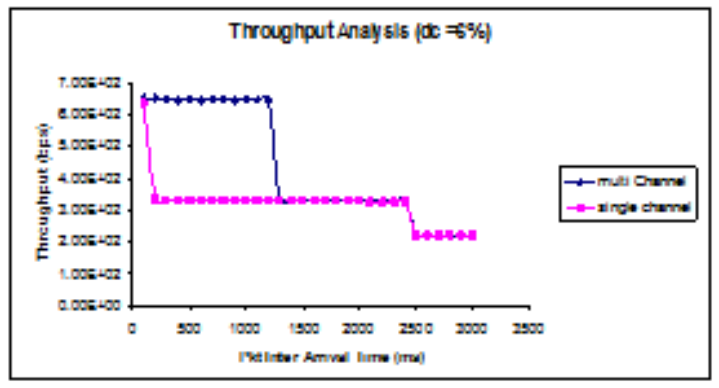

(b)

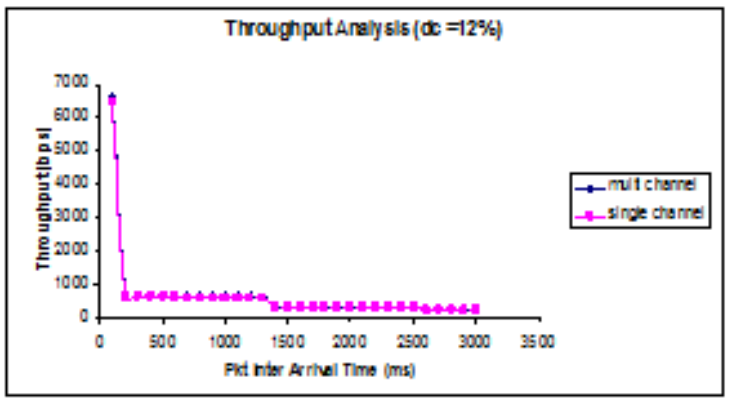

(d)

Figure 12. Duty Cycled Multi Channel MAC - Throughput Analysis (a) Duty Cycle 4\%

(b) Duty Cycle 6\% (c) Duty Cycle 10\% (d) Duty Cycle 12\%

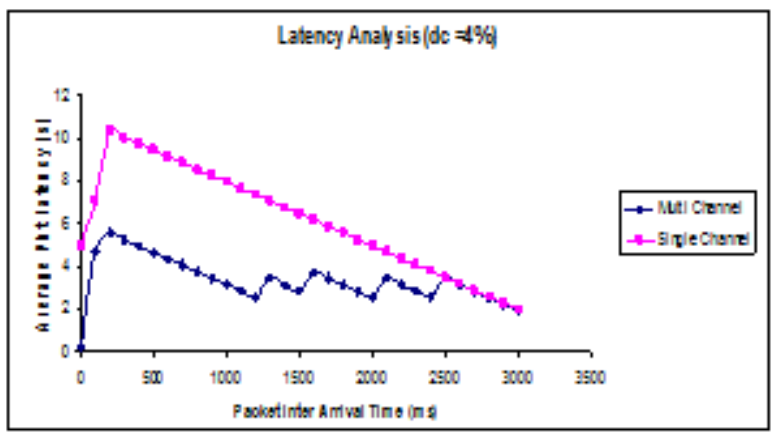

(a)

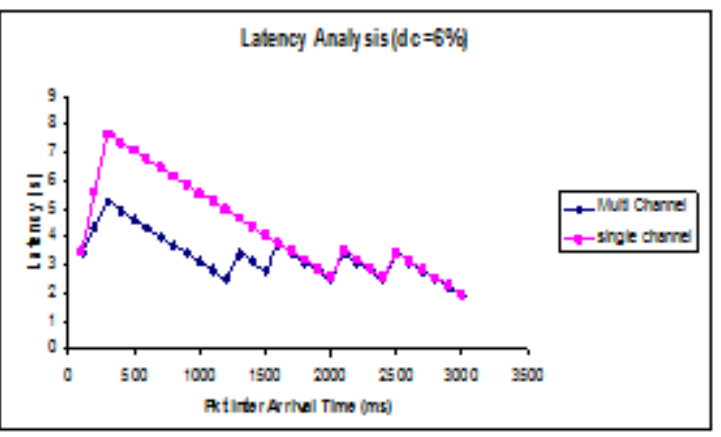

(b) 


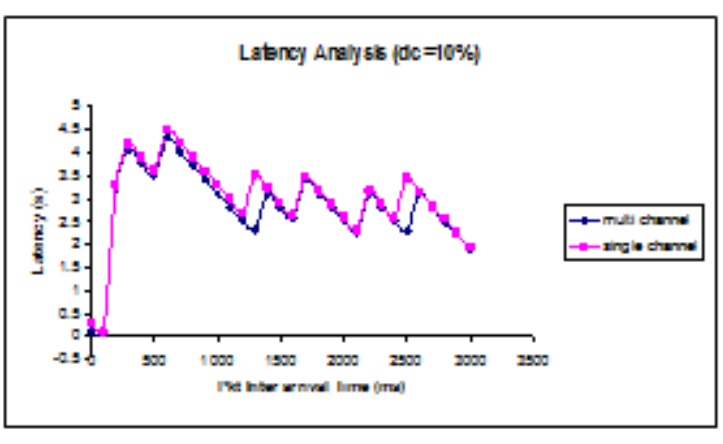

(c)

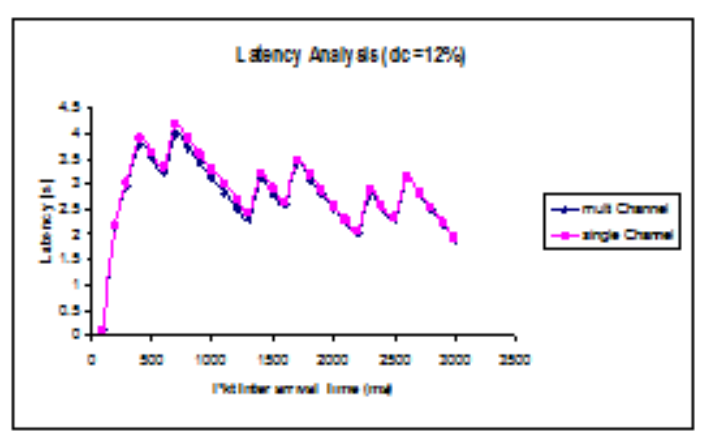

(d)

Figure 13. Duty Cycled SMC MAC - Latency Analysis (a) Duty Cycle 4\% (b) Duty Cycle 6\% (c) Duty Cycle 10\% (d) Duty Cycle 12\%

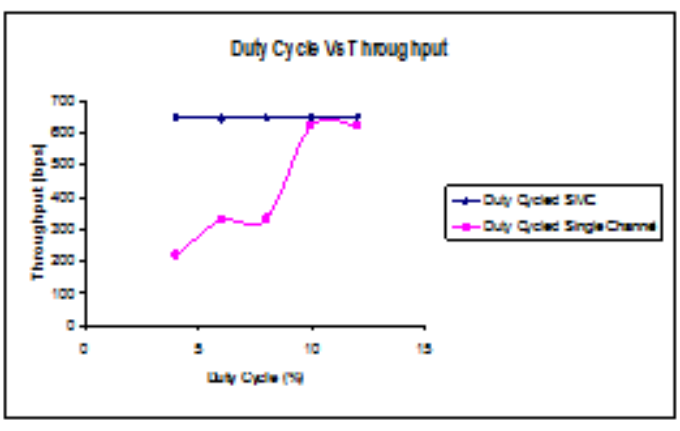

(a)

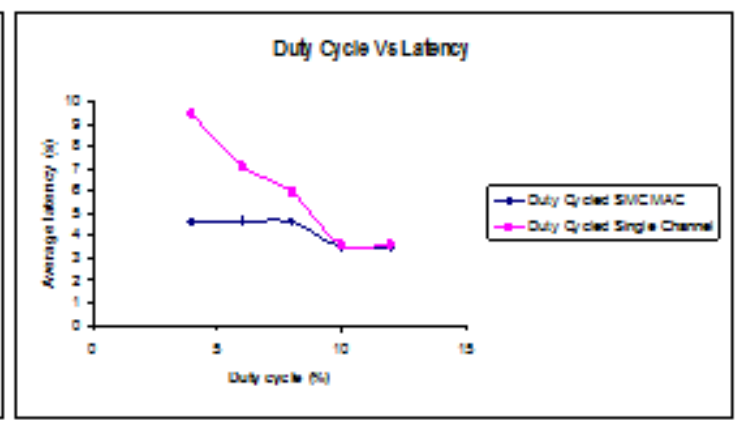

(b)

Figure 14.Effect of Duty Cycle in Protocol Performance (a) Duty Cycle Vs Throughput (a) Duty Cycle Vs Average latency

\section{CONCLUSION}

In this paper Duty Cycled Sensor Multi Channel (DC-SMC) MAC has been proposed for wireless sensor network. The proposed multi channel protocol uses a dedicated control channel and eight data channels. The contribution of the paper is it combines the status based channel assignment and measurement based channel information retrieval. The paper proposes the scheme to alleviate the issues in multi channel MAC like multi channel hidden terminal problem and the missing receiver problem. By taking the throughput and latency as the performance metric, the performance of DC-SMC MAC has been compared with that of the single channel CSMA/CA with RTS/CTS for various traffic loads. It is observed that the performance of DC-SMC MAC outperforms the single channel MAC in the high traffic conditions. It has been shown that, with the DC-SMC MAC, a higher throughput and lower latency can be achieved with minimal energy consumption. 


\section{REFERENCES}

[1] Jain N., Das S. and Nasipuri A. (2001), 'A multichannel CSMA MAC protocol with receiver-based channel selection for multihop wireless networks', in Proceedings of the 10th International Conference on Computer Communications and Networks, Phoenix, pp. 432-439.

[2] Li J., Haas Z.J., Sheng M. and Chen Y. (2003), 'Performance evaluation of modified IEEE 802.11 MAC for multi-channel multi-hop ad hoc network', in AINA 2003: Proceedings of the 17th International Conference on Advanced Information Networking and Applications, China, pp. 312317.

[3] So J. and Vaidya N.H. (2004), 'Multi-channel mac for ad hoc networks: handling multi-channel hidden terminals using a single transceiver', in MobiHoc '04: Proceedings of the 5th ACM international symposium on Mobile ad hoc networking and computing, ACM Press, New York, NY, USA, pp. 222-233.

[4] Chen J., Sheu S. and Yang C. (2003), 'A new multi channel access protocol for IEEE 802.11 ad hoc wireless LANs', in PIMRC 2003: The Proceedings of the 14th IEEE Personal, Indoor and Mobile Radio Communications Symposium, Vol. 3, pp. 2291-2296.

[5] Alnifie G. and Simon R. (2007), 'A multi-channel defense against jamming attacks in wireless sensor networks', in Q2SWinet '07: Proceedings of the 3rd ACM workshop on QoS and security for wireless and mobile networks, New York, NY, USA, ACM, ISBN 978-1-59593-806-0, pp. 95-104.

[6] Wood A.D., Stankovic J.A. and Zhou G. (2007), 'Deejam: Defeating energy-efficient jamming in IEEE 802.15.4-based wireless networks', in Proceedings of the 4th Annual IEEE Communications Society Conference on Sensor, Mesh and Ad Hoc Communications and Networks (SECON '07), pp. $60-69$.

[7] Xu W., Trappe W. and Zhang Y. (2007), 'Channel surfing: defending wireless sensor networks from interference', in IPSN '07: Proceedings of the 6th international conference on Information processing in sensor networks, New York, NY, USA, pp. 499-508.

[8] Kim Y., Shin H. and Cha H. (2008), 'Y-MAC: An Energy- Efficient Multi-channel MAC Protocol for Dense Wireless Sensor Networks', in Proceedings of International Conference on Information Processing in Sensor Networks (IPSN'08), Missouri, USA, pp. 53-63.

[9] Mastooreh S., Hamed S. and Antonis K. (2007), 'HYMAC: Hybrid TDMA/FDMA Medium Access Control Protocol for Wireless Sensor networks', in PIMRC 2007: The proceedings of the 18th IEEE Personal, Indoor and Mobile Radio Communications Symposium, Athens, Greece, pp. 1-5.

[10] Milica Jovanovic D. and Goran Lj. Djordjevic (2006), 'TFMAC: Multi-channel MAC Protocol for Wireless Sensor Networks', in Proceedings of 8th International Conference on Telecommunications in Modern Satellite, Cable and Broadcasting Services (TELSIKS 2007), pp-23-26.

[11] Gang Zhou, Chengdu Huang, Ting Yan, Tian He, John A. Stankovic and Tarek F. Abdelzaher (2006), 'MMSN: Multi-frequency media access control for wireless sensor networks', in Proceedings of IEEE International Conference on Computer Communications, Barcelona, Spain, pp.1-13

[12] Jingpu Shi, Theodoros Salonidis and Edward W. Knightly (2006), 'Starvation mitigation through multichannel coordination in CSMA multi-hop wireless networks', Proceedings of the 7th ACM International Symposium on Mobile Ad hoc Networking and Computing, pp. 214-225.

[13] Jiandong Li, Zygmunt J. Haas, Min Sheng and Yanhui Chen (2003), 'Performance Evaluation of Modified IEEE 802.11 MAC for Multi-Channel Multi-Hop Ad Hoc Network', in Proceedings of the International Conference on Advanced Information Networking and Applications (AINA 2003), pp. 312-317.

[14] Luo T., Motani M. and Srinivasan V. (2006), 'CAMMAC: A Cooperative Asynchronous MultiChannel MAC Protocol for Ad Hoc Networks', in Proceedings of IEEE Third International Conference. Broadband Communications, Networks and Systems (BROADNETS '06), San Jose, CA, pp.1-10.

[15] Priyank Porwal and Maria Papadopouli (2004), 'On demand channel switching for multi-channel wireless MAC protocols', Proceedings of 12th European Wireless Conference, Athens, Greece, [online] http://ics.forth.gr/netlab/mobile/publications/ew06.pdf. 
[16] Asis Nasipuri and Samir R Das (2000), 'Multi Channel CSMA with signal power based channel selection for multihop wireless networks', Proceedings of Vehicular Technology Conference, Vol.1, pp. 211-218.

\section{AUTHOR}

M. Ramakrishnan was born on $9^{\text {th }}$ May 1980 in Thirunelveli District, Tamil Nadu, India. He has completed his Bachelor's degree in Electrical and Electronics Engineering from the University of Madras in 2001 and has completed his Master of Engineering degree from Faculty of Electrical Engineering, Anna University, Chennai in 2005. He pursued his $\mathrm{PhD}$ studies in Wireless Sensor Networks as a UGC Research Fellow since 2007. After his $\mathrm{PhD}$, he has worked as Chief Technology Officer in Reindeer Technologies Private Ltd, Chennai for 4 years and currently he is working as an Associate Professor in

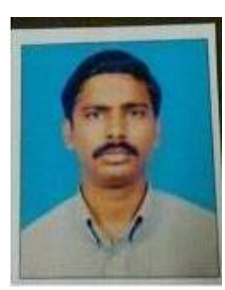
Department of Electrical and Electronics Engineering of Vel Tech Dr.Rangarajan and Dr.Shakunthala Technical University. He has 2 years of teaching experience. His research interests are protocol development for wireless sensor networks, embedded systems and signal processing. 